\title{
VON DER ARBEIT AM FILM
}

\author{
Die österreichische Amateurfilmkultur \\ der Zwischenkriegszeit
}

1 Bisherige Recherchen zeigen, dass vor allem die frühe österreichische Amateurfilmpraxis eine vorwiegend männliche Domäne war; der Unterstrich soll einerseits den geschlechtsspezifischen Charakter der Filmpraxis betonen und andererseits den (wenigen) Filmamateurinnen den ihnen zustehenden Raum geben.

2 Das Forschungsprojekt "Doing Amateur Film. Soziale und ästhetische Praktiken im österreichischen Amateurfilm der 1920er-bis 1980er-Jahre» wird von Sarah Lauß, Michaela Scharf und Sandra Ladwig in Kooperation mit der Universität für angewandte Kunst Wien, dem Österreichischen Filmmuseum und dem Ludwig Boltzmann Institut für Geschichte und Gesellschaft realisiert und von der Österreichischen Akademie der Wissenschaften im Rahmen eines Doc-team-Stipendiums (Okt. 2016-Sept. 2019) gefördert.
Dampfende Waggons verfolgen mechanisch geleitet ihren vorbestimmten Weg, vorerst auf Schienen und schließlich an massiven Stahlbaukonstruktionen hängend. Nun werden die Kohleladungen ausgekippt und weiterbefördert innerhalb des gigantischen Gaswerkes, über das der Filmamateur Friedrich Kuplent in seinem «Industriefilm» Gas (AT I933) berichtet. Die zwischen diesen Transportprozessen vereinzelt arbeitenden Menschen verlieren sich in den Totalaufnahmen und doch sind sie es, welche die Abläufe erst ermöglichen. Auch die Episode von der Gasverwendung in verschiedenen anderen Fabriken und Betrieben (Großwäscherei, Schlachterei, Großküche, Druckerei) zeigt die technischen und somatischen Vorgänge aus zurückhaltender Distanz. Die Werktätigen verblassen zu Miniaturen der Schaltstellen zwischen den rauchenden Maschinen und dem Produkt, zu anonymen Rädchen im Getriebe. Kuplents Film ist ein seltenes Beispiel dafür, dass sich Filmamateur_innen in der Zwischenkriegszeit das Sujet der klassen- und existenzbestimmenden Lohnarbeit und deren Alltag im (sub)urbanen Raum zu eigen machen. ${ }^{1}$

Erste Beobachtungen und Sichtungen im Amateurfilmbestand des Österreichischen Filmmuseums (ÖFM) ${ }^{2}$ verdeutlichen, dass der eigene Arbeitsplatz von Amateur_innen nur sehr vereinzelt im Register der gewählten Filmstoffe auszumachen ist. Klassenzugehörigkeit begründet sich somit in der frühen Amateurfilmkultur weniger über sichtbare Produktionsverhältnisse respektive Lohn- und Reproduktionsarbeit. Vielmehr verkörpern, visualisieren und rhythmisieren Amateurfilme Erfahrungen der Freizeit. Die Amateur_innen weisen durch ihre Arbeit am Film Freizeitkultur als Ort der Aushandlung, der Bewusstseinsbildung von Klasse aus. Im Folgenden wird diese Filmtätigkeit in Dialog mit der zeitgenössischen Ratgeberliteratur als kulturelle Praxis nachgezeichnet. Der Schwerpunkt liegt dabei auf den Klubfilmer_innen, die sich in ihrer Organisationsform von den Familienfilmer_innen deutlich 
unterscheiden. Die Organisierung und Strukturierung des Filmens selbst, so meine These, bestätigt den Bezug auf <bürgerliche> Wertvorstellungen als (Rettungs-)Versuch der Identitäts- und Standortbestimmung in der von erodierenden Klassengegensätzen geprägten Zwischenkriegszeit.

\section{Vom Filmen in der Freizeit}

«As things are, Spare Time is a time when

we have a chance to do

what we like, a chance to be most ourselves.» ${ }^{3}$

Mit zunehmender Freizeit entwickelt sich dieser Lebensbereich spätestens seit dem Ende des Ersten Weltkriegs zu einem gesellschaftlich wie individuell relevanten Erfahrungsraum, der politisch und moralisch aufgeladen - von christlich-sozialen über völkisch-nationalistische bis hin zu sozialistischen Diskursen - verhandelt wird. Die Einrichtung einer demokratischen Republik, die wirtschaftlichen Entwicklungen mit zunehmend komplexen hierarchischen Abhängigkeitsverhältnissen und Veränderungen hin zu einer rationalisierten Produktionsweise, die Aufhebung des Adels, die Entstehung einer «industriellen Reservearmee der Angestellten», ${ }^{4}$ die Herausbildung massenkultureller Freizeitangebote ebenso wie die erstarkende Arbeiter_innen- und Frauenbewegung stellen das Bürgertum als starre, undurchlässige Klasse in Frage. In der Freizeitkultur finden diese Erschütterungen und (Neu-)Konfigurationen ihren Niederschlag insofern, als dass Klassenverhältnisse sozial differenzierter, ambivalenter und dynamischer in Erscheinung treten. Über neu entstehende Aktivitäten und Konsumkulturen segmentieren sich (geschlechts- wie klassenspezifische) Vorstellungen von und Erfahrungen in der Freizeit, die von produktiver Selbstverwirklichung und -optimierung über Bildungs- und Reproduktionsarbeit bis zur Erholung und Entspannung reichen. ${ }^{5}$ Organisiertes Klubfilmen scheint im Rahmen dieser einschneidenden gesellschaftlichen Transformationen im Produktions- und Reproduktionsbereich Vorstellungen von einer bürgerlichen Lebensform zuzuarbeiten, wobei die (verschollene) bürgerliche Klasse restituiert werden soll. ${ }^{6}$

Anhand der Kategorien Home Movie (Familienfilm) und Klubfilm lassen sich zwar die heterogenen Amateurfilmpraktiken differenzieren; diese sind jedoch in der konkreten Filmpraxis häufig durchlässig: Während es etwa äußerst engagierte und ambitionierte Familienfilmer_innen gab, die aufwendige Spielfilme produzierten, verfolgten manche Filmklubmitglieder kaum ihre filmische Weiterentwicklung. Was Amateurfilmpraktiken unterscheidet, ist nicht nur ihr sozialer Rezeptionskontext, sondern auch die Produktionsästhetik: Während die einen mit der Kamera vor allem aufzeichneten und dokumentierten, also nichtfiktionale Filmchroniken produzierten, nutzten andere die Möglichkeiten des Films als Erzählmittel und Experimentierwerkzeug. ${ }^{7}$
3 Voice-over-Kommentar in dem Dokumentarfilm: Spare Time, Regie: Humphrey Jennings, UK 1939.

4 Siegfried Kracauer: Die Angestellten. Aus dem neuesten Deutschland, Frankfurt/M. 2016, 13.

5 Chris Rojek: Decentring Leisure. Rethinking Leisure Theory, London 1999; ders.: Capitalism and Leisure Theory, London 2014 [1985].

6 Vgl. zu der Lebenshaltung und den Kulturbedürfnissen der Angestellten: Kracauer: Die Angestellten, $92 \mathrm{ff}$.

7 Dabei überlagern sich zuweilen auch die schöpferische Nutzung des Mediums und registrierende Aufzeichnung: Vgl. Charles Tepperman: Amateur Cinema. The Rise of North American Moviemaking, 1923-1960, Oakland 2015, 169-192. 


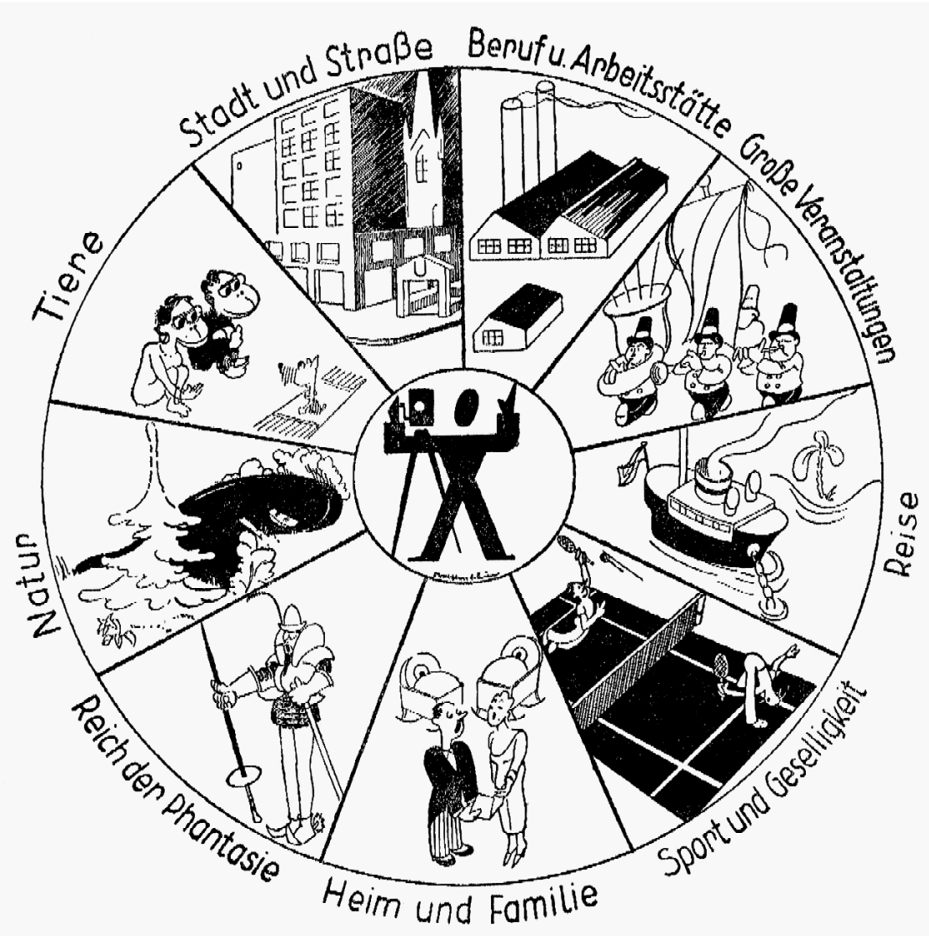

Im Home Movie wird über «kinematografische Fehler» oder einen fehlenden «roten Faden» hinweggesehen, weil die Evokation gemeinsamer wie individueller Erinnerungen für die Beteiligten zentral ist. $^{8}$ Die häufig inkohärente, fragmentarische Narration und «mangelhafte» Gestaltung zeugt davon, dass sich der Familienfilm üblicherweise nicht am industriellen Filmschaffen orientiert, da gemeinsame Erlebnisse und Lebensgeschichten im Vordergrund stehen. Der performative Akt der Familienfilmpraxis stellt Film und nicht zuletzt Familie (wieder) her und spiegelt gewissermaßen das privatisierte und emotionalisierte Familienkonzept als tragendes bürgerliches Leitbild in der Gesellschaft wider. ${ }^{9}$ Wiederkehrende Darstellungen von der

Abb. 1 In der Ratgeberliteratur galt das Motiv der (Lohn-)Arbeit durchaus als «filmenswert»

8 Vgl. Roger Odin: Reflections on the Family Home Movie as Document. A Semio-Pragmatic Approach, in: Patricia R. Zimmermann: Mining the Home Movie. Excavations in Histories and Memories, Berkeley 2008, 255-271.

9 Vgl. Alexandra Schneider: Die Stars sind wir. Heimkino als filmische Praxis, Marburg 2004, $65 \mathrm{ff}$.

$10 \mathrm{Vgl}$. Andreas Gestrich: Geschichte der Familie im 19. und 20. Jahrhundert, Berlin, Boston 2013, $4 \mathrm{ff}$., $36 \mathrm{ff}$. 11 Vgl. Odin: Reflections, $61 \mathrm{f}$.

12 Schneider: Die Stars sind wir, 11. In einigen Amateurfilmen aus der NS-Zeit manifestiert dagegen der darin visualisierte Reichsarbeitsdienst die (propagierte) Aufhebung der Klassengesellschaft in der antisemitischen, ‘produktiven Volksgemeinschaft?. Vgl. Willi Nißler: Wir haben gelernt!, in: Der Kino-Amateur, Nr. 8, H. 3, 1. März 1935, 58 ff. fürsorglichen und liebevollen Mutter, Erzieherin und Ehefrau verweisen dabei zum einen auf die geschlechtsspezifische Rollenverteilung und zum anderen auf die polarisierten Geschlechtscharaktere, die das uneingelöste Gleichheitsversprechen der Aufklärung in Bezug auf die Geschlechter markieren. ${ }^{10}$

Familienfilme reproduzieren nicht nur aufgrund ihrer stereotypen Gestaltung familiale Ideologie, auch das Ausgesparte konstruiert die trügerischen Erzählungen, die sich vorwiegend als Antithesen zum Alltag darstellen." In eskapistischer Manier wird ein Leben frei von ökonomischen Zwängen imaginiert: Das Gewohnte und Belastende ist im Familienfilm zensuriert, viel eher thematisiert er die «Idylle des modernen bürgerlichen Lebens, voller Freude und Glück».12 Was den kleinen Film, den Schmalfilm, vom klassischen Erzählkino, dem dokumentarischen Film und der Filmkunst abhebt, ist, dass selbst bei vorausgehender Planung Erleben und Dokumentation zusammenfallen: Fiktion, Vorstellungskraft und Wirklichkeit verbinden sich im Amateurfilm zu einer schwer entwirrbaren Erzählung davon, wie es gewesen sein könnte. ${ }^{13}$

Anders als die Familienfilmer_innen beziehen sich die Klubfilmer_innen auf den professionellen und kommerziellen Film. Der institutionelle Rahmen einer Film-Gemeinschaft impliziert zudem einen halböffentlichen Vorführkontext. Insbesondere Klubfilmer_innen befinden sich in einem hoch organisierten, künstlerischen «Regime», ${ }^{14}$ die Mitglieder streben «höhere» Ziele 
als «Gelegenheitsoperateure», Erinnerungs- und Familienfilmer_innen an. Scheint die Familienfilmpraxis überwiegend einem individuellen oder sehr begrenzten gemeinschaftlichen sozialen Bedürfnis nach bewegter Erinnerung $\mathrm{zu}$ entspringen und in eben dieser Erinnerungszusammenkunft aufzugehen, so vollzieht sich in der Klubfilmaktivität die Wandlung vom Zeitvertreib zum Wettkampf, der ein weitaus regelkonformeres und planvolleres Handeln einfordert. ${ }^{15}$ Klubangehörige sind wettbewerbsorientiert, wollen die eigene Leistung vorführen und weiterentwickeln, wie sich bei den folgenden Ausführungen zum Klub der Kinoamateure Österreichs (KdKÖ) zeigen wird.

\section{Der KIub der Kinoamateure Österreichs (KdKÖ) ${ }^{16}$}

Der Produktionsästhetik eines Amateurfilms ist die anvisierte Aufführung meist schon eingeschrieben, wobei sich das Publikum je nach Gattung hochgradig unterscheidet. Die Filme wurden tendenziell nicht für die Projektion im öffentlichen Lichtspielhaus produziert, jedoch zeigt ein Blick in die Geschichte des KdKÖ, dass die nichtprofessionelle Filmtätigkeit kein ausschließlich privates Vergnügen war. ${ }^{17}$ Auch wenn Familienfilme bei Wettbewerben eingereicht und im Verein gezeigt wurden, kritisieren die Mitglieder des Klubs in ihren Mitteilungen 1935 eine Vermengung beider Amateurfilmpraktiken: «Die für den Autor sicherlich höchst wertvollen Familienbilder bleiben für den Unbeteiligten gänzlich nichtssagend und wirken geradezu störend: ein Beispiel für die Zwiespältigkeit des Amateurs.»18

Handbücher und Zeitschriften entwerfen Bilder von und für Amateur_innen, die spezifisch bürgerliche Werte vermitteln: Die Anstrengungen der ernsthaften, lernenden und «vorwärtsstrebenden» Filmer_innen, über den Familienkreis hinauszuwachsen, werden schließlich von Erfolg gekrönt sein. ${ }^{19}$ Organisierte Klubangehörige wetteifern innerhalb anerkannter Genres wie Spielfilm, Reportage oder Reisebericht in nationalen wie internationalen Konkurrenzen um eine gute Platzierung und orientieren sich offensichtlich an Standards der professionellen Filmproduktion. Aus diesen spezifischen Kennzeichen der Klubkultur ergeben sich folgende Überlegungen: Gerade weil sich Angehörige des Filmklubs an der Schnittstelle von öffentlich und privat bewegen, lastet auf ihren Aktivitäten ein Legitimationsdruck, dem mit sozialer Distinktion gegenüber dem Familienfilm begegnet wird. Dabei sind die ästhetischen Praktiken der Filmenden im Klub ständig unter Leistungs- und Beweiszwang, was auch einer bürgerlichen Konzeption von Arbeit entspricht. ${ }^{20} \mathrm{Be}-$ wertungsmaßstäbe, die vom industriellen (und mitunter vom experimentellen) Film ausgehen, leiten und beeinflussen das Selbstverständnis des Klubs und somit auch die filmischen Arbeiten der Amateur_innen. In ihre Konzeption von Freizeit ist das Arbeitsethos als grundlegende und notwendige Voraussetzung integriert, als Arbeit an sich selbst und als Arbeit an der (Film-)Form, wobei die Einhaltung der Regeln des Filmsportes eine Steigerung des Erfolges und
13 Vgl. zu den Filmformaten 35 , 16, 9, 5, $8 \mathrm{~mm}$ : Hellmuth Lange:

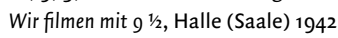
(Filmbücher für alle 8), 9-13.

14 Ob Ryan Shand mit diesem Begriff «nur das System an Gestaltungsnormen im Filmklub beschreibt oder auch auf Jacques Rancières Ansatz des ästhetischen Regimes verweist, bleibt unklar. Vgl. Ryan Shand: Theorizing Amateur Cinema. Limitations and Possibilities, in: The Moving Image. The Journal of the Association of Moving Image Archivists, Vol. 8, Nr. 2, 2008, 36-6o, hier 54.

15 Vgl. Roger Caillois: Die Spiele und die Menschen. Maske und Rausch, Stuttgart 1960, 37 ff.; Alexandra Schneider, Wanda Strauven: Filmspielerei. Digitale Home Movies von Kindern, in: Ute Holfelder, Klaus Schönberger (Hg.): Bewegtbilder und Alltagskultur(en). Von Super 8 über Video zum Handyfilm. Praktiken von Amateuren im Prozess der gesellschaftlichen Ästhetisierung, Köln 2017, 203-221.

16 Frühe Mitglieder des KdKÖ bildeten keine homogene sozioökonomische Klasse, entstammten aber tendenziell den oberen Einkommensgruppen.

17 Vgl. dazu die Urania-Vorführungen des KdKÖ: Aus den Vereinen. Klub der Kinoamateure Österreichs, Wien 3., in: Der Kino-Amateur. Offizielles Organ des Klubs der Kino-Amateure Österreichs, Sitz Wien, Nr. 7, H. 3 , 1. März 1934, 92.

18 Der Kino-Amateur. Offizielles Organ des Klubs der Kino-Amateure Österreichs, Sitz Wien, Nr. 8, H. 5 , 1. Mai $1935,146$.

19 Vgl. Alex Strasser: Filmentwurf, Filmregie, Filmschnitt. Gesetze und Beispiele, Halle (Saale) 1937 (Filmbücher für alle 3), 12 ff.; Friedrich Kuplent: Vom Schnitt, vom Kleben und der Montage des Films. Winke für Kino-Amateure aller Formate, in: Der Kino-Amateur, Jg. 6, Nr. 2, 1. Februar 1933, 37-41.

20 Vgl. Bernhard Schäfers: Die bürgerliche Gesellschaft. Vom revolutionären bürgerlichen Subjekt zur Bürgergesellschaft, Wiesbaden 2017, 17 ff.; Rojek: Capitalism and Leisure Theory, 61-74; ders.: Decentring Leisure, 45-47. 
21 Vgl. Christa Reim: Erziehung zum filmischen Sehen, in: Der Kino-Amateur, Nr. 11, H. 10, Oktober 1938, 268; Strasser: Filmentwurf, Filmregie, Filmschnitt, 13.

22 Vgl. Hermann Bausinger: Bürgerlichkeit und Kultur, in: Jürgen Kocka (Hg.): Bürger und Bürgerlichkeit im 19. Jahrhundert, Göttingen 1987 , 121-142, hier $121 \mathrm{f}$.

23 Vgl. Siegfried Kracauer: 104. Die Biographie als neubürgerliche Kunstform, in: ders.: Aufsätze 1927-1931, Schriften 5, 2, Frankfurt/ M. 1990, 195-199, hier $195 \mathrm{ff}$.

24 Vgl. Carl M. Kotlik: Die Amateurkinobewegung in Österreich, in: Der Österreichische Amateur-Filmer, Nr. 1, März 1934, 4-7. Vgl. dazu auch: N. N.: Kinoamateure sind Freunde des Films, in: Der Wiener Film, Nr. 1, H. 17, 1. September 1936, 3. Unter österreichischer Mitwirkung konstituiert sich 1931 die UNICA (Union Internationale du Cinéma d'Amateur) als internationaler Zusammenschluss, von dem bis heute Wettbewerbe und Zusammenkünfte organisiert werden.

25 Hanns Plaumann: Die Amateurfilmbewegung in Europa, in: Der Kino-Amateur, Nr. 8, H. 10, 1. Oktober 1935, 265-268, hier 268.

26 Hans Innerhofer: Der Kinoamateur am Scheideweg, in: Der Kino-Amateur, Nr. 9, H. 11, 1. November 1936, 297-300, hier $298 \mathrm{ff}$.

27 Ebd., 300. des ästhetischen Genusses verspricht. ${ }^{21}$ Die Freizeit der Amateur_innen - und damit ihre ästhetischen Praktiken - unterstehen gewissermaßen einer zweckmäßigen und rationalisierten Lebensform, die nun jene Vorstellungen implementiert, die zuvor auf die Arbeitswelt bezogen waren: Bildung, individuelle Leistung und Aufstiegsstreben. Der Lebensbereich der Lohnarbeit weist einen zunehmend verschwindenden klassenspezifischen, eigenständigen Bestand auf (in der Betriebshierarchie sind alle formell gleich Lohnabhängige), während sich in der Freizeit eine durch gemeinsame Werte und Leitbilder begründete Freizeit- bzw. Amateurfilmkultur konstituiert. Die «bürgerliche Kultur» kann dabei als Ensemble von Werten und Verhaltensstilen gefasst werden, die jedoch schon im Laufe des I9. Jahrhunderts zunehmend klassenübergreifend wirksam sind. ${ }^{22}$ Tendenziell verleiht organisiertes Filmen in der Freizeit einem in Bedrängnis geratenen bürgerlichen Individuum nun Bedeutung und Spielraum, indem es seine unsicher gewordene Existenz über die Vereinsgemeinschaft und mittels einer kultivierten Freizeitaktivität stabilisiert. ${ }^{23}$

Mit der zunehmenden Institutionalisierung in den I93oer Jahren wächst allmählich die Amateurfilmbewegung: Der Gründer des KdKÖ Carl M. Kotlik sieht Amateurfilm als (noch nicht entsprechend anerkannten) Sport, wichtigen Kulturfaktor und Mittel zur «Völkerverständigung»; durch den internationalen Austausch wird der nationalen Bewegung zugearbeitet als auch dem_der Einzelnen Geltung verschafft. ${ }^{24}$ Konkurrenz und Wettbewerb basieren im Filmklub auf der durch (Selbst-)Disziplin hergestellten Leistung und Anerkennung des Individuums durch die anderen Angehörigen der Gemeinschaft. Die Mitgliedschaft im Klub diene der «gedeihliche[n] Fortentwicklung der Arbeit des einzelnen», wobei vermehrt an die künstlerische Leidenschaft der Filmamateur_innen appelliert wird..$^{25}$

Das KdKÖ-Mitglied Hans Innerhofer erläutert den Wert individueller Tatkraft wie folgt:

\footnotetext{
Nicht der Besitz einer raffinierten Spezialkamera, sondern nur eine besondere schöpferische Kraft hebt uns aus der Masse empor. [...] Hier stehe ich und ich muß von diesem Standorte meine eigene Individualität fixieren. [...] Nicht nur der gute Einfall, sondern erst die Umsetzung in die filmische Tat zeigt den Meister. [...] Was uns Österreicher im besonderen Maße aber verpflichtet, ist unser künstlerisches Ansehen in der ganzen Welt. ${ }^{26}$
}

Hier wird deutlich, dass Kreativität noch anderen, höheren Zielen genügen soll als nur dem, die eigene Individualität und ästhetische Artikulation auszubilden, schließlich steht die nationale Reputation auf dem Spiel. Der individuelle Ausdruck, so Innerhofer, diene vor allem der Repräsentation Österreichs. Es gälte den Amateurfilm als verhältnismäßig junge Ausdrucksform «im Dienste unserer Heimat» mitzuprägen. ${ }^{27}$ Das organisierte Filmen in der Freizeit entspringt einem übergeordneten kulturellen Geltungsbedürfnis, aus dem schließlich auch die Bedeutung für das Individuum resultiert. Zusammenfassend lässt sich der 
KdKÖ als nationaler Zusammenschluss ausmachen, der sich, von preisgekrönten Amateur_innen des Klubs vertreten, in der internationalen Konkurrenz behaupten soll; appelliert wird hier an eine Amateur_innenidentität, die in der ambivalenten Spannung zur Vereinsgemeinschaft hervorsticht und in dieser Verbindung an einer nur vage definierten, gesellschaftlichen Entwicklung des Films teilhat. Die Veröffentlichungen des KdKÖ in der Zwischenkriegszeit zeigen, dass schöpferische Individualität sich durch den Bezug auf Kulturansprüche von Verein und Nation vollzieht und damit zugleich ein bürgerliches Konzept beschreibt: Kreative Persönlichkeit wird wechselseitig durch den übergeordneten Anspruch genährt und bringt diesen damit wiederum hervor. ${ }^{28}$

\section{Klasse und Amateurfilmpraktiken}

Die Masse der Angestellten unterscheidet sich vom Arbeiter-Proletariat darin, dass sie geistig obdachlos ist. Zu den Genossen kann sie vorläufig nicht hinfinden, und das Haus der bürgerlichen Begriffe und Gefühle, das sie bewohnt hat, ist eingestürzt, weil ihm durch die wirtschaftliche Entwicklung die Fundamente entzogen worden sind. Sie lebt gegenwärtig ohne eine Lehre, zu der sie aufblicken, ohne ein Ziel, das sie erfragen könnte. Also lebt sie in Furcht davor, aufzublicken und sich bis zum Ende durchzufragen. ${ }^{29}$

Die Beschäftigung mit historischem Amateurfilmmaterial erfordert auch eine Auseinandersetzung mit der gesellschaftlichen und individuellen Konzeption von Freizeit. Wie diese Zeit organisiert wird, ist keineswegs eine freie Entscheidung, sondern von klassenspezifischen Vorstellungen und Hierarchien geprägt. ${ }^{30}$ Neben diesen Zeitstrukturen beeinflusst die selbstbestimmte Praxis des (Amateur-)Filmens vor allem die «Ästhetik der Ökonomie» ${ }^{31}$ hinsichtlich einer «effizienten» Gestaltung und Zeitordnung, aber auch in Bezug auf die notwendige Auswahl aufzeichnungswürdiger Momente des Lebens. Amateurfilm(en) ist ein exklusives und kostspieliges Vergnügen, das jedoch denen, die es sich leisten konnten, verschiedenste technische Möglichkeiten bietet, von der Herstellung einfacher «lebender Familienchroniken» ${ }^{32}$ bis hin $\mathrm{zu}$ aufwendigeren Produktionen mit Trickaufnahmen. Die Klubfilmkultur erfordert und verbindet ökonomisches (sich das Filmen leisten zu können) und soziales Kapital (Mitgliedschaft/Gruppenzugehörigkeit) sowie objektiviertes (Amateurfilmapparatur) und inkorporiertes kulturelles Kapital (angeeignete Kenntnisse im Umgang mit Kamera-, Montage-, Projektionstechniken). ${ }^{33}$ Frühe Amateurfilmproduktionen werden nicht nur aufgrund der hohen Anschaffungs- und Materialkosten zumeist sorgfältig geplant. Der Umstand, dass belichtetes Filmmaterial weder gelöscht noch überschrieben werden kann, setzt ebenfalls Kalkül voraus, was das Amateurfilmschaffen (im Unterschied zu heutigen medialen Praktiken) maßgeblich beeinflusst. Eher Maschine denn Werkzeug, erfordert die Handhabung der Amateurfilmapparatur Training wie strukturierte Beschäftigung. Die Kontrolle, die bei handwerklichen Tätigkeiten noch prägend war, erodiert bei der
28 Vgl. zur Ausrichtung der bürgerlichen Familie an den Werten der Nation: Schäfers: Die bürgerliche Gesellschaft, 24.

29 Kracauer zum «Asyl der Obdachlosen" in: Die Angestellten, 91.

30 Vgl. Zu Haus- und Theoriearbeit von Frauen: Heike Klippel: Zeit ohne Ende. Essays über Zeit, Frauen und Kino, Frankfurt/M., Basel 2009, 94-132. Zu Max Weber: Rojek: Capitalism and Leisure Theory, 61-74. 31 Mark Neumann: Amateur Film. Automobility and Cinematic Aesthetics of Leisure, in: Laura Rascaroli, Barry Monahan, Gwenda Young (Hg.): Amateur Filmmaking. The Home Movie, the Archive, the Web, New York 2014, 51-64, hier 52.

32 Wolfgang Jaensch: Hallo! Sie filmen noch nicht? Kurzgefasste Anleitung für Amateur-Kinematographie, Berlin 1928, 8.

33 Vgl. Pierre Bourdieu: Ökonomisches Kapital - kulturelles Kapital - soziales Kapital, in: Dorothee Kimmich, Schamma Schahadat, Thomas Hauschild (Hg.): Kulturtheorie, Bielefeld 2010, 271-286, hier 275-279. 
34 Vgl. Vrääth Öhner: Spezialisierte Fragmentierung. Zu den technischen Bedingungen der Einbildungskraft im frühen Amateurfilm, in: Zeitschrift für Kulturwissenschaften, Nr. 2: Vorstellungskraft, hg. v. Siegfried Mattl, Christian Schulte, 2014, 51-6o, hier $54 \mathrm{ff}$.

$35 \mathrm{Vgl}$. Siegfried Kracauer: Theorie des Films. Die Errettung der äußeren Wirklichkeit, Frankfurt/M. 2015, 41.

36 Vgl. Aleida Assmann: Ist die Zeit aus den Fugen? Aufstieg und Fall des Zeitregimes der Moderne, München 2013, $34 \mathrm{ff}$.

37 Hans Carl Opfermann: Das Filmen ist so schön, Halle (Saale) 1938,55 . industriellen ebenso wie bei der nichtindustriellen Filmproduktion durch die Komplexität der Maschine. ${ }^{34}$ Bei aller Beschränkung und Voraussetzung erlauben jedoch die ästhetischen Praktiken des Filmens die reale, standardisierte Zeit und somit auch die erlebte Freizeit neu zu ordnen, umzuorganisieren und zu rhythmisieren, um letztlich von ihr zu erzählen, ob im ausgearbeiteten Spielfilm oder im fragmentarischen Urlaubsfilm der Familie. Amateurfilmer_innen vergewissern sich des eigenen Sehens und hinterlassen folglich eine Setzung, die das Geschehen vor der Kamera und den Akt des Sehens fixiert. Der Film verwandelt nicht nur die dreidimensionale materielle Wirklichkeit in eine zweidimensionale Fläche, sondern im aufzeichnenden Vorgang des Sehens findet, wie von Siegfried Kracauer beschrieben, zugleich eine (subjektiv) ordnende und gliedernde Tätigkeit statt. Filmen ermöglichte den frühen Amateur_innen mittels der Arbeit am Film, sich selbst ins Bild zu setzen und die materielle Wirklichkeit auf das für sie Wesentliche zu reduzieren oder sogar in das Unwirkliche und Unvorstellbare vorzudringen. Ähnlich den Fotograf_innen sehen auch Amateurfilmer_innen die Dinge in und mit ihrer Seele. ${ }^{35}$ Amateurfilmen erfüllt, formt und fokussiert das Zeiterleben und ermöglicht - im Gegensatz zu anderen Freizeitpraktiken - den Rückgriff auf die moments of being, die sich durch Differenz zur Routine und zum Alltag definieren. ${ }^{36}$

In den I93oer Jahren schreibt der Handbuchautor Hans Carl Opfermann zur Differenz zwischen Filmen als Lohnarbeit und dem «Kinosport»: «Wir haben dem Berufsmann jedenfalls etwas voraus, was für den Enderfolg ausschlaggebend ist: Unsere große Liebe und unermüdliche Begeisterung zum Filmen, die ungetrübt vom Zwang des täglichen Broterwerbs der Freude unserer freien Stunden dienen darf. ${ }^{37}$ Opfermann stellt die Begeisterung und Liebe zum Filmen dem Zwang des Broterwerbs noch gegenüber, doch in der Praxis der frühen, organisierten Amateur_innen wird der fremdbestimmte Zwang bereits überführt in ein selbstgewähltes Verlangen nach Verbesserung, nach Optimierung der Filmtätigkeit, die $<$ Erfolg $>$ verspricht. Die Strukturierung und Organisierung des Film(en)s im Klub führt Arbeit in diese Freizeit- und Medienpraxis ein, wodurch reines Aufzeichnen zugunsten von Gestaltung überwunden werden soll. Während für Familienfilmer_innen tendenziell das Filmen in nichts an Arbeit erinnern soll, setzen die Klubfilmer_innen diese unter anderen Vorzeichen fort. Ob es an der technischen Schwierigkeit von Innenaufnahmen liegt, dass Amateurfilmer_innen selten die eigene Stellung im Produktionsprozess visualisierten, oder der mit Arbeitsteilung und Spezialisierung einhergehende Entfremdungsprozess (vom Produkt ihrer Arbeit wie von der Tätigkeit) dazu beigetragen hat, kann wohl nicht eindeutig geklärt werden. Wenn Entfremdung der hauptsächliche Grund für die auffällige Abwesenheit von Lohnarbeit in Amateurfilmen ist, so kann die Freizeit als jener Bereich der Kompensation ausgemacht werden, dem nun das zugeschrieben wird, was die Abhängigkeit von und Verankerung in scheinbar verselbstständigten ökonomischen Verhältnissen verhindert: eine selbstbestimmte Tätigkeit. Die Filmklubaktivität überführt das entfremdete und 
mit Auflösung konfrontierte (in dieser Zeit fast ausschließlich männliche) Individuum in eine Sphäre, die es ihm erlaubt, eigenständig an einer Gemeinschaft zu partizipieren, welche einmal national, ein anderes Mal international argumentierend Selbstverwirklichung über das Filmen fordert und fördert. Nicht nur ist die (freie und schöpferische) Entfaltung des Selbst in der Vereinsgemeinschaft in hohem Maße von der Anerkennung durch andere abhängig und reglementiert. Auch verdeutlicht sich die trügerische Erwartung, Freizeit als jenen (produktiv) zu füllenden Raum so strukturieren und organisieren zu können, dass die im Verschwinden begriffene bürgerliche Klasse durch die in ihr verfochtenen Werte restituiert werde. ${ }^{38}$

In den Diskursen zum Amateurfilm wird der Begriff des Sportes mitsamt den sozialen Implikationen von Erfolg und Misserfolg in die Konzeption dieser Freizeitbeschäftigung integriert. Die Ambition zum Filmen ist spätestens mit der Organisierung im Filmklub als ein Messen der Fähigkeiten zu definieren, das in den Wettbewerben der Filmklubs seine radikalste Zuspitzung erfährt. Die fachliche Resonanz und der soziale (auch klubübergreifende) Zuspruch ist zentraler Aspekt bei der Produktivität der Klubfilmer_innen, sodass die Medienpraxis der frühen Amateur_innen zwischen Freiheit und Erwartungsdruck, künstlerischem Ausdruckswollen und rigiden Gestaltungsnormen, die ihm erst gültige Form geben sollen, oszilliert. Der Legitimationsdruck, dem sich frühe Amateur_innen ausgesetzt sehen, fördert Strukturen wie Wettbewerbsdenken, Leistungsprinzip und Individualismus, die das verhältnismäßig freie Schaffen, zwar nicht in ökonomische, doch aber in Verwertungslogiken drängt.

Die vermeintliche Freiheit von ökonomischen Zwängen bei der Produktion eines Amateurfilms stellt sich, nicht zuletzt wegen der damit verbundenen Ausgaben, als eine eingeschränkte dar; auch führen Formen der Anerkennung (symbolisch, sozial wie auch finanziell) im Rahmen von Amateurfilmwettbewerben die Abstraktion des Tauschprinzips wieder ein. ${ }^{39}$ Der Wert des Films wird im Agon des Amateurfilms bestimmt und gegen Anerkennung, gegen Preisauszeichnungen getauscht. Auch wenn Amateurfilme im konkurrierenden Klubwesen keine Warenförmigkeit im ökonomischen Sinne annehmen, so finden trotzdem Prozesse des Tausches, der Wertbestimmung statt. Diese Arbeitsstrukturen von Filmklubs können als Reproduktionszyklen verstanden werden, die sich laut Heike Klippel strukturell und funktional durch ihren produktiven Überschuss auszeichnen: Gewesenes und Anerkanntes wird erhalten (das erreichte Niveau der Amateur_innen und die Orientierung an Standards der professionellen und kommerziellen Filmproduktion), jedoch tritt an die Stelle des Alten und Singulären nun ein Neues, Nichtidentisches (die ästhetischen Formen des Filmtextes) und ermöglicht somit Wandlung und Fortschritt der strukturellen Entwicklung des Klubs. ${ }^{40}$ Festgestellt wird immer wieder ein beachtliches Niveau, auf welchem die vor allem an Wettbewerben teilnehmenden Amateur_innen sich bewegen. Der Maßstab ist hierbei ausgerichtet an zeitgenössischen professionellen Filmformen (Reportage, Spielfilm, Reisefilm,
38 Vgl. Kracauer: Die Angestellten, $81 \mathrm{f}$.

39 Hier knüpfe ich an Überlegungen von Siegfried Mattl und Vrääth Öhner an, vgl. dies.: Ästhetik des Möglichen. ¿Der grüne Kakadu» als Bricolage heterogener Traditionen, in: Siegfried Mattl, Carina Lesky, Vrääth Ohner, Ingo Zechner (Hg.): Abenteuer Alltag. Zur Archäologie des Amateurfilms, Wien 2015, 71-86, hier $76 \mathrm{f}$.

40 Klippel: Zeit ohne Ende, 14-21. 

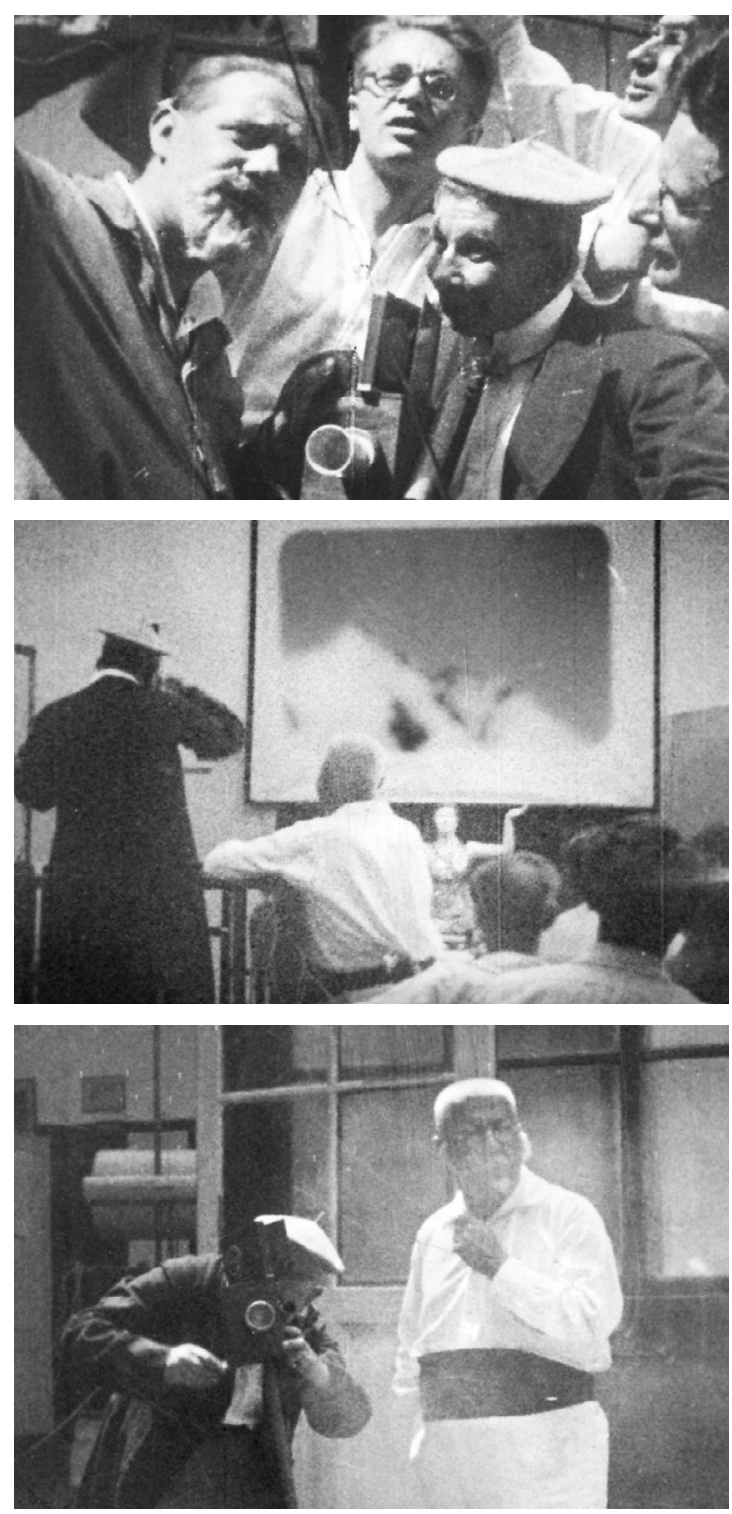

Abb. 2-4 Screenshots aus: Ludi als Kinoamateur, Regie: Friedrich Kuplent, 9,5 mm, AT r $93^{\circ}$, stumm, 9 Min. In den bürgerlichen> Amateurfilmpraktiken des KdKÖ finden sich mitunter ambivalente Strukturen: Die Filmgroteske unterminiert als Gemeinschafts-/Vereinsfilm gewissermaßen das bürgerliche, individualistische Kulturverständnis des Klubs abstrakter Film) und verweist mithin auf ein Moment der Reproduktion, das dem Prozess der Filmherstellung der Klubamateur_innen inhärent ist.

Die Orientierung an diesen etablierten Formen fördert ein dynamisches Fortbestehen der Erzähl- und Gestaltungsweisen des <großen> Films im Amateurklubwesen - dynamisch deshalb, weil Amateurfilmpraktiken durch ihre Verschiedenheit vom professionellen Bereich diesen Formen immer wieder ein produktives Neues, Anderes, Eigenes hinzufügen (sowohl formal durch Format oder Montage als auch inhaltlich durch Sujet oder Narration oder durch eine kollektive Arbeitsweise). Aufgrund ihres vielfältigen kulturellen Gebrauchs nimmt Amateurkinematografie nicht zwangsläufig eine oppositionelle oder nachahmende Haltung zu professionellem oder künstlerischem Filmschaffen ein, vielmehr positioniert sie sich als Parallelkultur. ${ }^{41}$ Die Abweichung ist dabei weniger als programmatische Differenz zu verstehen, sondern speist sich aus den bastelfreudigen Arbeitsweisen der frühen Amateurfilmkultur, aus der Bricolage. Die ästhetischen Praktiken des KdKÖ unterminieren jedoch - entgegen der bisher konturierten <bürgerlichen> Arbeit am Film - mitunter bürgerliche Wertvorstellungen: Erfindungsreichtum und Experimentierfreudigkeit, spielerische und kreative Verwirklichung von Ideen und kollektive Produktionsstrategien betonen, dass die Strukturierung von Freizeit auch jenseits eines zweckrationalen Handelns liegen kann.

Leider sind bisher kaum Amateurfilme aus der Zwischenkriegszeit ausfindig gemacht worden, die gesichert Arbeiter_innen zugeordnet werden können; die wenigen Aufnahmen von Maifeiern und anderen Massenereignissen in der Zwischenkriegszeit könnten ebenso als Auftragsarbeiten der Partei entstanden sein. ${ }^{42}$ Die Grenzen der Produktions- und Verwendungsweisen scheinen insbesondere in diesem Bereich zwischen Amateurfilm, (semi-)professionellem Film, Dokumentar-, Werbe- und Propagandafilm fließend zu sein. Der Bund der sozialistischen Arbeiterfilmer, von dem bisher nur paper trails nachgewiesen sind, positioniert sich Anfang der i93oer Jahre entgegen der skizzierten Linie des KdKÖ: Hier wird weniger Individualismus gefördert als vielmehr das Privileg Filmen nun für (alle) Arbeiter_innen zugänglich gemacht: 
Wenn auch normalerweise das Selbstaufnehmen von Kinofilmen nicht eben wohlfeil ist, so ist es doch das Verdienst des Bundes, das Filmen für seine Mitglieder derartig verbilligt zu haben, daß man ruhig sagen kann: Filmen ist nicht teurer als photographieren! Die gemeinsame Materialbeschaffung, vollständige Umstellung auf Selbstausarbeitung, gemeinsamer Besitz von Arbeitsgeräten und Maschinen und sorgfältige Schulung haben es ermöglicht, zu diesem Resultat zu kommen. ${ }^{\mathbf{4 3}}$

Zwei Parallelen lassen sich in den Diskursen dieser beiden Zweige des Amateurfilms erkennen: Sowohl der bürgerliche KdKÖ als auch der Bund der sozialistischen Arbeiterfilmer erachteten technische Kenntnisse für eine schöpferische Filmtätigkeit als notwendig und griffen zudem ähnliche Genres, wie Natur- und Sportaufnahmen, Ausflugsfilme oder Reportagen, auf. Was aber die beabsichtigte Wirkung und übergeordnete Nutzung betrifft, so sind die Ziele unterschiedlich: Der Bund der sozialistischen Arbeiterfilmer versteht Amateurkinematografie nicht nur als persönliche Liebhaberei, sondern als Kampfmittel im Dienste des Sozialismus. ${ }^{44}$ Die Filme sollten die Wirklichkeit zeigen und im Interesse der Arbeiter_innenschaft sein. Die Schriften der sozialistischen Bildungszentrale und des Bundes der sozialistischen Arbeiterfilmer lassen - nach anfänglicher Skepsis gegenüber dem neuen Medium Anfang der I93oer Jahre - Vorstellungen vom Film als Propagandamittel und Medium der Aufklärungsarbeit erkennen, das vom «schlechten» Unterhaltungsfilm und von bürgerlichen Vergnügungspraktiken distanziert schließlich seiner sozialen Bestimmung, dem politischen (Klassen-)Kampf, zugeführt werden müsse. ${ }^{45}$ Der zu erobernde Filmsport sollte als «echter Arbeitersport» die kollektive und kollektivierte Arbeit am Film in den Dienst der politischen Aufklärung stellen. Die Vorspiegelung einer Scheinwirklichkeit war zentraler Kritikpunkt an der bürgerlichen Kultur, der eine realistische Darstellung des Arbeiter innenlebens, der «Wirklichkeit», vorgezogen wurde. Ein Vergleich mit der Arbeiter_innenfotografie der I92oer und I93oer Jahre zeigt, dass der geforderten Bildsprache des «sozialen Sinns», dem kollektiven Einsatz der Kamera als erzieherische, scharfe geistige Waffe im Kampfe des Proletariats nicht nachgekommen, sondern viel eher Motive der bürgerlichen Welt - einer Kultur des Besitzes und individualistischen Persönlichkeitskultur - mitgeschleppt wurden: Die Fotografien bezeugen eine (verständliche) Flucht vor dem Alltag und der sozialen Wirklichkeit, die sich somit kaum von bürgerlichen Lichtbildern unterscheiden. ${ }^{46}$

Die organisierten <bürgerlichen> Amateur_innen suchten in ihrer Freizeit sich selbst vergewissernd und verwirklichend, kompetitiv, nachahmend, eskapistisch, selten experimentierend und manchmal humoristisch ihren Weg als Parallelkultur zwischen industriellem Kino und Filmkunst. Über privatistische Belange hinaus sollte an einer gesellschaftlichen Filmkultur partizipiert werden, wobei grundlegende politische, soziale oder ästhetische Transformationen durch die Vereinigung der Interessen im Klub und die Arbeit am Film kaum angestrebt wurden. Der Blick ist wiederholt auf das vom professionellen Kino
41 Vgl. Öhner: Spezialisierte Fragmentierung, 57; Shand: Theorizing Amateur Cinema, $55 \mathrm{ff}$.

42 Vgl. Ludwig Boltzmann Institut für Geschichte und Gesellschaft (Hg.): Stadtfilm Wien. Die große Demonstration der Wiener Arbeiterschaft am 12. November 1927 , http:||stadtfilm-wien.at/film/136|, geehen am 19.4.2018.

43 Die Jahresversammlung der Arbeiterfilmer, in: Arbeiter-Zeitung, Nr. 45, H. 185, 5. Juli 1932, 8.

44 Vgl. Arbeiter-Zeitung, Nr. 45, H. 44 , 13. Februar 1932, 8. Sowie: Das Kleine Blatt, Nr. 6, H. 180, 30. Juni 1932, 10.

45 Vgl. JosefWeidenholzer: Auf dem Weg zum iNeuen Menschen. Bildungs- und Kulturarbeit der österreichischen Sozialdemokratie in der Ersten Republik, Wien 1981, $207 \mathrm{ff}$.

46 Vgl. Anton Holzer: Vorwärts! Die österreichische Arbeiterfotografie der Zwischenkriegszeit, in: Fotogeschichte, Beiträge zur Geschichte und Ästhetik der Fotografie, Nr. 33 , H. 127, 2013, 17-30, hier 22. Nach Hermann Bausinger verweist die wechselseitige Bezugnahme zum einen auf die ‘Kulturfähigkeit` der Arbeiter_innen und zum anderen auf den in der bürgerlichen Aufklärung entwickelten Gedanken der klassenübergreifenden Bildsamkeit. Vgl. Bausinger: Bürgerlichkeit und Kultur, $133 \mathrm{f}$. 
Erreichte und Etablierte gerichtet, was einer Identität der Amateurfilmbewegung ebenso zuarbeitete wie der Leistung des Individuums. Frühe Amateurfilmpraktiken sind von einer arbeitsfunktionalen Logik geprägt, die sie ebenso aufgreifen wie durchkreuzen.

Trotz aller Abgrenzungsversuche weisen die ästhetischen Praktiken der Arbeiter_innen und des KdKÖ ambivalente Durchlässigkeit auf: Während zumindest für die proletarische Fotografie das Übernehmen bürgerlicher Motive geltend gemacht werden kann, so nutzten <bürgerliche〉 Filmamateur_innen kollektive Produktionsstrategien, um das Gemeinschaftsgefühl im Verein zu stärken. Einerseits wurde die Unabhängigkeit von ökonomischen Zwängen gegenüber dem Berufsfilm betont, andererseits schien der radikale Regelbruch in Form einer eigenständig entwickelten, emanzipierten Amateurfilmkultur kaum möglich, womit sich die Amateurfilmpraktiken in der Zwischenkriegszeit als Freizeitkultur zu erkennen geben, in der die Auseinandersetzungen um klassenspezifische Wertvorstellungen und Standortbestimmungen anhand des Film(en)s noch ausgetragen werden. 